\title{
Evaluating Energy Consumption of Proactive and Reactive Routing Protocols in a MANET*
}

\author{
Marco Fotino ${ }^{1}$, Antonio Gozzi ${ }^{1}$, Juan-Carlos Cano $^{2}$, Carlos Calafate ${ }^{2}$, Floriano \\ De Rango ${ }^{1}$, Pietro Manzoni ${ }^{2}$, and Salvatore Marano ${ }^{1}$ \\ 1 University of Calabria \\ Pietro Bucci, 87036 Rende, ITALY \\ Email: \{fotino, gozziantonio\}@gmail.com \\ \{derango, marano\}@deis.unical.it \\ 2 Polytechnic University of Valencia \\ Camino de Vera, s/n, 46071 Valencia, SPAIN \\ Email: \{jucano, calafate, pmanzoni\}@disca.upv.es
}

\begin{abstract}
Ad hoc routing technology has been developed primarily for networks of mobile nodes. The operational life of untethered nodes will be limited by its power source, so ad hoc networks strongly depend on the efficient use of their batteries. All the layers of communication are coupled in power consumption and solutions addressing the power saving issue include transmission power control, power aware routing and low power modes at the physical layer. At the network layer, routing protocols may balance power consumption at nodes according to their routing decisions. Several authors have proposed power aware routing algorithms based on power cost functions. In this work we present a performance comparison of the DSR (Dynamic Source Routing) and the OLSR (Optimized Link State Routing) in terms of energy consumption. We evaluate how the different approaches affect the energy usage of mobile devices. We found that a reactive protocol takes advantage of its routing policy when the traffic load is low. However, at higher traffic rates, a proactive routing protocol can perform better with an appropriate refresh parameter. In addition, we showed how, independently from the routing protocol selected, the overhearing activity can seriously affect the performance since all the neighbours of a transmitting node also consume their energy. To the best of our knowledge, this is the first simulation study addressing the power saving issue to extensively compare the DSR and OLSR protocols under a wide variety of network scenarios.
\end{abstract}

\section{Introduction}

In the last few years, thanks to the proliferation of wireless devices, the use of mobile networks is growing very fast. In particular, a very large number of recent studies focuses on Mobile Ad-hoc Networks, also known as MANETs [1].

\footnotetext{
* This work was partially supported by the Ministerio de Educación y Ciencia, Spain, under Grant TIN2005-07705-C02-01. and by the Generalitat Valenciana, Ayudas complementarias para proyectos de $I+D+i$, Spain, under Grant ACOMP07/237.
} 
A MANET is a network without a fixed infrastructure, in which every node can act as a router; this is required when the two end-points interchanging data are not directly within their radio range. This kind of network, self-organizing and self-reconfiguring, is very useful when it is not economically practical or physically possible to provide a wired networking infrastructure (battlefield scenarios, natural disasters, opportunistic networks etc.). Performance of a mobile ad hoc network depends heavily on the selected routing scheme, and the traditional Internet routing protocols do not work efficiently in a MANET. This kind of network, in fact, has a dynamic topology (every node can move randomly and the radio propagation conditions change rapidly over the time) and a limited bandwidth (so that the control traffic overhead must be reduced to the minimum) [2]. Developing routing protocols for MANETs has been an extensive research area in recent years, and many proactive and reactive protocols have been proposed from a variety of perspectives ([3]-[8]). These protocols try to satisfy various properties, like: distributed implementation, efficient bandwidth utilization, throughput optimization, fast route convergence and freedom from loops. Since mobile hosts today are powered by battery, efficient utilization of battery energy is a key factor. When a node exhausts its available energy, it ceases to function and the lack of mobile hosts can result in partitioning of the network, thereby affecting the overall communication performance.

The key to energy efficiency in future wireless terminals will be at the higher levels: low-energy protocols, energy-cognisant user interfaces, context dependent, and predictive shutdown management will be used to reduce the computation done at the terminal. The networked operation of a wireless terminal opens up additional techniques for increasing energy efficiency. Each protocol layer can use its own power conservation scheme. Electronically, we can turn off or slow down some devices (CPU, disk, antenna) when not needed, or adjust transmission power according to the interference level. At the data-link layer, energy conservation can be achieved using effective retransmission request schemes and powering off the nodes not participating in a communication in order to avoid the overhearing problem. At the network layer, we can modify routing metrics to prefer routes requiring lower levels of transmission power. These approaches can however affect other classical metrics such as the end-to-end delay and throughput, but it is a must to extend the network's lifetime.

In this work we measure and compare the energy consumption behaviour of two routing protocols: the Dynamic Source Routing (DSR) [9], which follows a reactive approach, and the Optimized Link State Routing (OLSR) [10], which uses a proactive one. We pursue a double objective. Firstly, we want to evaluate how different approaches affect the energy usage of mobile devices when using two of the most promising routing protocols currently considered under IETF's MANET working group [1]. In fact, among the great variety of different proposals, DSR and OLSR have arrive to the RFC status. Secondly, we want to check whether or not, under the IEEE 802.11 technology, some of the power aware routing proposals in the literature could be efficiently utilized to extend the lifetime of nodes and connections. In fact, we believe that, because of the 
overhearing and idle activity of a network interface card based on the current IEEE 802.11 technology, a majority of the proposed schemes not only are quite tricky to be implemented, but also could not achieve their assumed benefits. The simulation results presented in this paper were obtained using the ns- 2 simulator [11], which is a discrete event, object oriented, simulator developed by the VINT project research group at the University of California at Berkeley.

This paper is organized as follows. In Section 2 we give a brief description of the routing protocols we compared, outlining their basic differences that could affect their energetic behavior. Section 3 describes the simulation environment and the energy model we implemented. Section 4 presents the obtained simulation results and finally, some concluding remarks are made in Section 5.

\section{Routing protocols for MANETs}

In this section we briefly review the main concepts dealing with the two protocols we analyzed, the DSR, and the OLSR respectively. This area is anyway a very dynamic one, and up-to-date information about the status of this area can be found in the web site of IETF's MANET group [1]. The MANET working group proposes two kinds of routing protocols: reactive and proactive. A reactive (or on-demand) routing protocol determines routes only when there is data to be sent. If a route is unknown the source node initiates a search to find one, and it is primarily interested in finding any route to the destination, not necessarily the optimal route. A proactive routing protocol, instead, attempts to continuously maintain routes to all destinations, regardless of whether they are required or not. To support this behaviour, the routing protocol propagates periodic information updates about network's topology or connectivity throughout the network. With respect to path selection, we can distinguish between source routing schemes, in which intermediate routers merely act as store-and-forward devices, sending the packet to the next hop according to the route indicated on the packet's header, and non-source routing systems. In the latter ones routers determine the path throughout the network based on their own calculations, assuming that hosts only have partial information about routes. Such routing protocols can be based on a link-state or a distance-vector algorithm. Traditionally, in a link-state algorithm each router floods routing information about the state of its own links to all nodes in the network, thereby building a picture of the entire network on its routing tables. On the other hand, in a distancevector algorithm each router sends all (or a part) of its routing table only to its neighbors. From the addressing point of view, we can have a hierarchical routing system (some routers form a sort of backbone) or a flat address space (where all routers are peers of all others).

\subsection{The DSR protocol}

The Dynamic Source Routing protocol (DSR) is a reactive protocol which tries to reduce the overhead, while providing a reliable routing sheme at the cost of 
not finding optimal routes. Mobile hosts don't rely on periodic messages, with a consequently energetic advantage in terms of battery consumption. DSR only updates its routes when it needs to react to link failures on the routes being used. The protocol is based on the use of two main mechanisms: Route Discovery and Route Maintenance, which work together to allow nodes to discover and maintain routes to arbitrary destinations in the ad hoc network. The protocol allows multiple routes to any destination and allows each sender to select and control the routes used when performing routing decisions. The DSR protocol also includes a guaranteed loop-free routing and a very rapid recovery when routes in the network change. The DSR protocol has been mainly designed for mobile ad hoc networks of up to about two hundred nodes, and is designed to work well even at very high rates of mobility.

\subsection{The OLSR protocol}

The Optimized Link State Routing (OLSR) proactive protocol is an optimization of the classical link state algorithm, tailored to the requirements of a MANET. Because of their quick convergence, link state algorithms are somewhat less prone to routing loops than distance vector algorithms, but they require more CPU power and memory. Proactive protocols can be more expensive to implement and support but are generally more scalable. The key concept used in OLSR is the multipoint relay (MPR). MPRs are selected nodes which forward broadcast messages during the flooding process. This technique substantially reduces the message overhead as compared to a classical flooding mechanism where every node retransmits each message received. This way a mobile host can reduce battery consumption. In OLSR, link state information is generated only by nodes elected as MPRs. An MPR node may choose to report only links between itself and its MPR selectors. Hence, contrarily to the classical link state algorithm, partial link state information is distributed in the network. This information is then used for route calculation. OLSR provides optimal routes in terms of number of hops. The protocol is particularly suitable for large and dense networks as the technique of MPRs works well in this scenarios.

\section{Simulation set-up}

The simulation results presented in this paper were obtained using the ns- 2 simulator. ns-2 is a discrete event, object oriented simulator developed by the VINT project research group at the University of California at Berkeley. The simulator has been extended to include: node mobility, a realistic physical layer that includes a radio propagation model, radio network interfaces and the IEEE 802.11 MAC protocol using the Distributed Coordination Function (DCF). The radio propagation model includes collisions, propagation delay and signal attenuation. In our experiments we have set a $54 \mathrm{Mbps}$ data rate, and a radio range of 250 meters. 


\subsection{Energy Consumption Model}

A generic expression to calculate the energy required to transmit packet $p$ is: $E(p)=i * v * t_{p}$ Joules, where: $i$ is the current consumption, $v$ is the voltage used, and $t_{p}$ the time required to transmit the packet. We suppose that all mobile devices are equipped with IEEE 802.11 g network interface cards (NICs). The energy consumption values were obtained by comparing commercial products with the experimental data reported in [12].

The values used for the voltage and the packet transmission time were: $v=$ $5 \mathrm{~V}$ and $t_{p}=\left(\frac{p_{h}}{6 * 10^{6}}+\frac{p_{d}}{54 * 10^{6}}\right) \mathrm{s}$, where $p_{h}$ and $p_{d}$ are the packet header and payload size in bits, respectively. We calculated the energy required to transmit and receive a packet $p$ by using: $E_{t x}(p)=280 m A * v * t_{p}$ and $E_{r x}(p)=240 m A *$ $v * t_{p}$, respectively. Since receiving a packet and just being idle, i.e., when simply powered on, are energetically similar [12], we assumed $E_{\text {idle }}(t)=240 m A * v * t$, where $t$ is the NIC idle time.

Moreover, we account for energy spent by nodes overhearing packets. As shown in [12], we assume the energy consumption caused by overhearing data transmission is the same as that consumed by actually receiving the packet.

For the purpose of evaluating the effect of overhearing, we modified the energy model to account not only for the energy expenditure due to transmission and reception, but also for overhearing packet exchanges. Thus, the total amount of energy, $E\left(n_{i}\right)$, consumed at a node $n_{i}$ is determined as:

$$
E\left(n_{i}\right)=E_{t x}\left(n_{i}\right)+E_{r x}\left(n_{i}\right)+E_{o}\left(n_{i}\right),
$$

where $E_{t x}, E_{r x}$, and $E_{o}$ denote the amount of energy expenditure by transmission, reception, and overhearing of a packet, respectively. Notice that, as the average number of neighboring nodes affected by a transmission increases, the network is more dense, and so Eq.(1) implies that the packet overhearing causes much more energy consumption.

\subsection{Methodology}

To compare the DSR and the OLSR protocols, we simulated a dense wireless network, with 50 nodes moving in a $870 \times 870 \mathrm{~m}$ area (with a density of about 66 nodes $/ \mathrm{km}^{2}$ ). Each node moves in this area according to the random waypoint mobility model, with a speed of $5 \mathrm{~m} / \mathrm{s}$ and no pause time. In terms of traffic there are $12 \mathrm{CBR} / \mathrm{UDP}$ sources generating 20 packets/s, packet size is set to 512 bytes. The duration of each simulation is 450 seconds, with a startup period during the first 15 seconds where no traffic is generated.

Due to the random nature of the mobility model we used, the results of each simulation were considered as IID random variables $(\mathrm{X} 1, \mathrm{X} 2, \mathrm{Xn})$ with finite mean. We repeated the simulations, i.e., we varied to the value of $n$, to obtain an estimation of with a 95 percent confidence interval, by using the following definition: 


$$
\bar{X}(n) \pm t_{n-1,0.95} \sqrt{\frac{S^{2}(n)}{n}}
$$

where $t_{n-1,0.95}$ is the upper 0.95 critical point for Student's $\mathrm{t}$ distribution with n-1 degrees of freedom, $X(n)$ is the sample mean and $S^{2}(n)$ is the sample variance.

We mainly analyzed the time when each node dies due to lack of remaining battery (i.e., expiration time of nodes) as well as the lifetime of connection which captures the effects of disconnections due to lack of possible routes (i.e., expiration time of connections). We also measured the average end-to-end delay per packet, as well as the throughput. Finally, we also study how NIC activities contribute to the total energy expenditure. For the purpose of investigating the effect of overhearing, and according to the energy model described earlier, we modified the ns-2 energy model to allow measuring the battery energy consumed when overhearing packet exchanges, as well as the energy due to the idle operation mode.

\section{Performance Study}

\subsection{Idle Power and Overhearing influence}

We first evaluate the influence of the Idle Power on the energetic consumption of mobile nodes. To do that we assign different costs to the Idle state: zero, $\frac{E_{r x}}{4}$, $\frac{E_{r x}}{2}$, and $E_{r x}$. All nodes have their initial energy values randomly selected, but in a range that avoids nodes with extremely low levels of energy, which might not even attempt to start communication.

Figure 1 shows the results obtained. We observe that, only in case of no idle power consumption, can we clearly see the difference between the reactive an the proactive protocols being tested. In the other cases, even with a low idle state energy consumption, all the nodes in the network tend to exhaust their battery at about the same time (i.e. when idle power consumes all the device energy), no matter whether we are evaluating DSR or OLSR.

In addition, for the purpose of investigating the effect of overhearing, we modified the energy model to allow the battery power to be consumed by overhearing packets in the wireless channel.

Figure 2 shows how many nodes have died over time due to lack of battery. We plot the results with and without considering the effects of overhearing. We can definitely observe different results between two cases. When we consider overhearing, all approaches behave similarly, because neighbors of a transmitting node consume considerable part of their energy overhearing transmissions. Each node spends a very large amount of energy to overhear packets addressed to other nodes. 


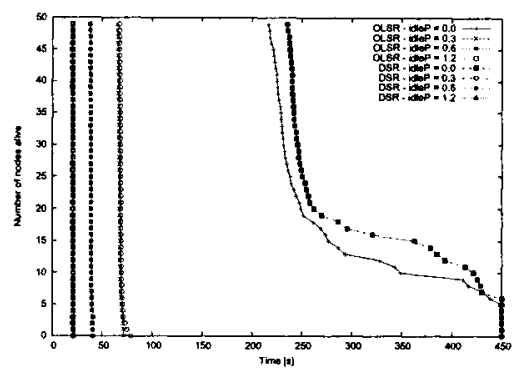

Fig. 1. Number of nodes alive vs time when varying idle power levels for the IEEE 802.11 techonolgy. $E_{r x}=1.2 \mathrm{~mW}$.

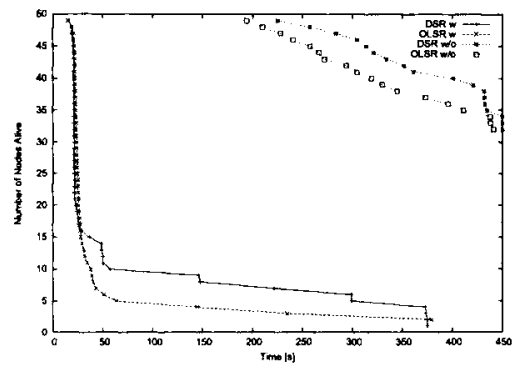

Fig. 2. Number of nodes alive vs time with (w) and without (w/o) considering overhearing.

We investigated the amount of energy consumed by participating nodes according to the network card activities. We observe that overhearing consumes most of the energy (see Figure 3). This implies that some techniques are required to reduce this energy expenditure by, for example, switching the network interface cards into the sleep mode.

Table 1 shows that the amount of energy spent in overhearing is larger than $90 \%$ for both protocols; which it mostly depends on node density and transmission range.

Table 1. Percentage of energy consumption: Transmission, Reception and Overhearing.

\begin{tabular}{|l|r|r|r|r|}
\hline & DSR w/o & OLSR w/o & DSR w & OLSR w \\
\hline Transmission & 63.32 & 65.60 & 3.84 & 4.10 \\
\hline Reception & 36.68 & 34.40 & 1.90 & 1.59 \\
\hline Overhearing & 0.00 & 0.00 & 94.27 & 94.31 \\
\hline
\end{tabular}




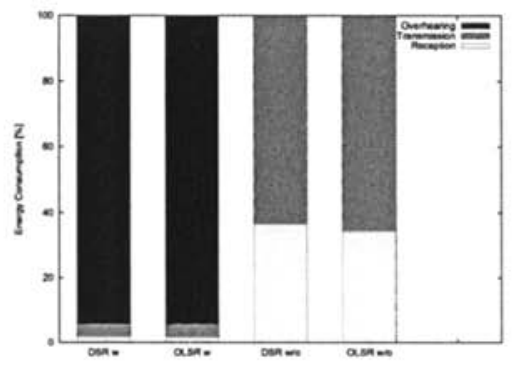

Fig. 3. Energy consumption(\%) with(w) and without(w/o) considering overhearing.

We conclude that the idle power and overhearing effects dominate the energy consumption in the simulation of a dense network. Given this result, it seems that improvements should focus on devices' hardware to reduce the energy due to the idle state and to overhearing by, for example, switching the network interface cards into the off state. Otherwise, with the current technology, no significant differences will appear with respect to the energy consumption among reactive and proactive routing protocols.

We are well aware that IEEE 802.11 network interface cards, in the near future, could reduce the energy consumption due to the idle state. Furthermore, they could also include new modes with low cost in terms of energy consumption and transmission time, which could reduce the overhearing activities. So, in the next sections, we consider an ideal futuristic NIC where the impact of the idle state is negligible, and concentrate our study on the differences between reactive and proactive approaches. We make tests on two different scenarios: a completely static environment and a dynamic one such as the one presented in Section 3.2.

\subsection{Static scenario}

We first evaluate the behaviour of DSR and OLSR when all nodes maintain their initial positions throughout the simulation. The network consist of 27 mobile nodes distributed over a $550 \times 750$ meters area. In this scenario, 12 sender nodes establish a CBR/UDP connection with 12 receivers; data is relayed by three forwarding nodes situated in the center. Each CBR connection generates a constant bit rate of 4 packets/second with a packet size of 512 bytes.

The sender nodes will mostly consume energy due to transmission activities. The receivers will spend a larger amount of energy in receiving, while forwarders must perform both tasks, exhausting their energy earlier.

Figure 4 left shows lifetime of connection. When using the DSR protocol the three forwarders nodes exhaust their battery simultaneously, so that all the connections expire at about the same time. When using the OLSR protocol, due to its proactive behaviour, there is a small gap between expiration times. However, even though the proactive protocol spends energy to build and update 
routing tables during the setup period (the first 15 seconds of simulation), it is able to maintain the connections alive for about the same time as the DSR protocol.
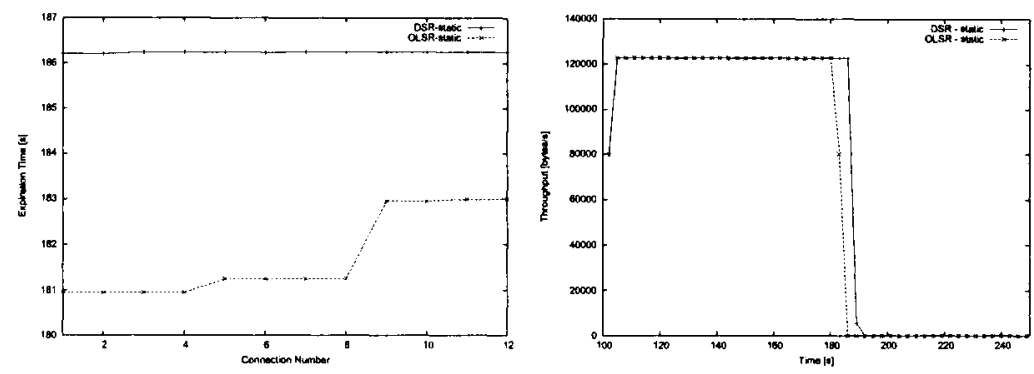

Fig. 4. Connections expiration (left) and throughput (right) in a static scenario.

Table 2 summarizes the performance of the protocols in the static scenario.

\begin{tabular}{|l|r|r|}
\hline & DSR & OLSR \\
\hline Data packet delivery ratio (\%) & 28.71 & 27.32 \\
\hline End-to-end delay (ms) & 3.31 & 2.63 \\
\hline Overhead (\%bytes) & 0.34 & 12.09 \\
\hline
\end{tabular}

Table 2. DSR - OLSR performance evaluation in a static scenario.

As for the end-to-end delay, it remains about $20 \%$ lower when using the OLSR protocol because the reactive DSR protocol must initially spend some time to find the path to the destination. With respect to the overhead, obviously it is higher when using the proactive approach. Finally, with respect to the data packet delivery ratio, the obtained results are almost the same independently of the selected protocol. The early exhaustion of the forwarding nodes' battery implies a network partition which seriously affects the packet delivery ratio. However, Figure 4 right shows that the throughput was maintained just until the network partition occurs.

\subsection{Dynamic scenario}

We now evaluate the impact of node mobility on performance. We simulated the DSR and the OLSR protocols in the dynamic scenario described in Section 3.2, and using the minimum hop count routing policy. Figure 5 shows the expiration time of nodes. We can observe how the DSR takes advantage from its reactive 
nature. During the first 15 seconds of simulation, while OLSR spends energy to update the network topology, DSR does not generate any packets. With respect to the lifetime of connections (see Figure 6 left) the response of OLSR and DSR is very similar, though obviously shifted since the proactive protocol starts its periodic exchange of message at the beginning of the simulation (15 seconds earlier).

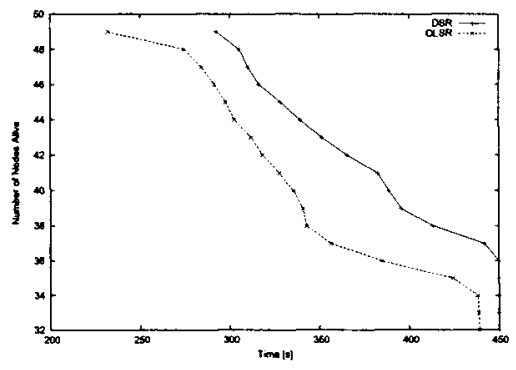

Fig. 5. Nodes alive vs time in a dynamic scenario.

Notice that, when compared to the static network, the lifetime of connections significantly increases. For the static network, some connections cannot progress when network partitioning occurs. However, for dynamic scenarios, node mobility favors the appearance of new paths after network partitioning disappears.
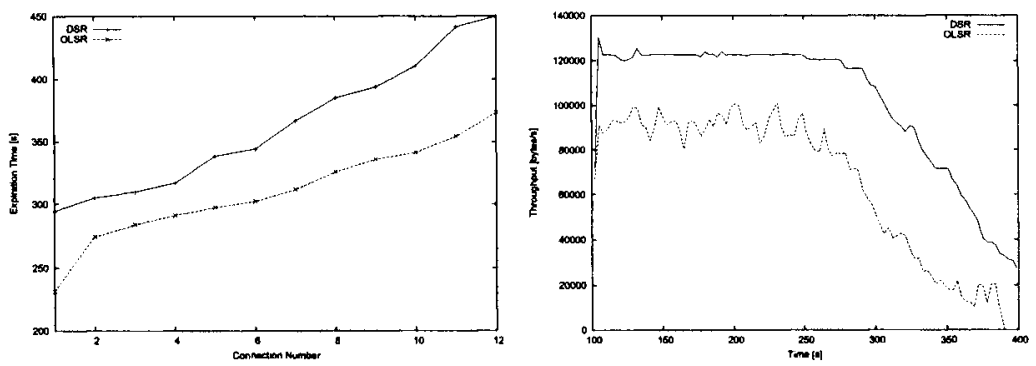

Fig. 6. Connections expiration (left) and throughput (right) in a dynamic scenario.

Table 3 summarizes the performance of the protocols in the dynamic scenario.

As expected, the DSR protocol performs better in terms of packet delivery ratio, end-to-end delay and routing overhead. The improvements in terms of routing overhead are expected since we are comparing reactive vs. proactive approaches. In terms of end-to-end delay notice that, with respect to the static 
Table 3. DSR - OLSR performance evaluation in a dynamic scenario.

\begin{tabular}{|l|r|r|}
\hline & DSR & OLSR \\
\hline Packet delivery ratio (\%) & 92.68 & 71.15 \\
\hline End-to-end delay (ms) & 12.23 & 13.54 \\
\hline Normalized routing overhead (\%bytes) & 0.86 & 14.77 \\
\hline
\end{tabular}

scenario, we now have longer routes (on average). Concerning the packet delivery ratio, Figure 6 right shows detailed results for throughput variations throughout time. Before to the expiration of connections, DSR has a very stable throughput, while the one offered by the OLSR varies a lot. In a dynamic scenario, the reactive nature of the DSR protocol allows it to rapidly reacts to path changes. When using the OLSR protocol, node mobility leads to frequent packet losses. This effect could be reduced by updating the routing tables of OLSR more frequently, but this could lead to very high values of routing overhead.

Finally, Figure 7 shows the offered throughput as the number of nodes have died over time. As expected, as the number of nodes in the network decreases, some connections can not go ahead, and the total aggregated throughput is reduced. Again, the observed differences between the two protocols comes from the packet losses affecting the OLSR due to node mobility.

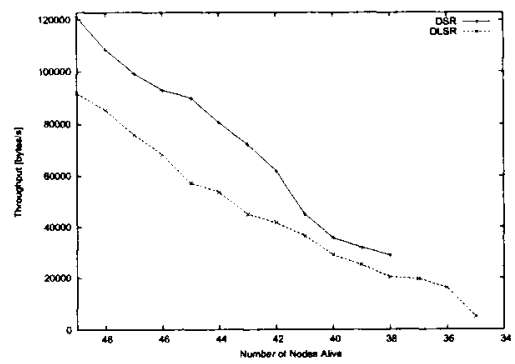

Fig. 7. Throughput vs number of nodes alive in a dynamic scenario.

\section{Conclusions}

In this work we analyze the energy consumption behaviour of the Dynamic Source Routing protocol and the Optimized Link State Routing protocol. We evaluate how different models for power consumption affect the energy usage of mobile devices. We found that a reactive protocol takes advantage from its routing policy when the traffic load is low. However, at higher traffic rates, a 
proactive routing protocol can perform well with an appropriate refresh parameter.

Extensive simulations under different MANE'T scenarios showed the importance of considering the overhearing and the idle mode as part of the energy model. We observe that, with the current technology, the idle power and overhearing effects dominate the energy consumption, and so new techniques should be investigated to reduce the energy consumption during idle periods due to overhearing.

The obtained results induce us to believe that some of the solutions found in the literature based on power-aware route selection metrics can not achieve their assumed benefits using real hardware. Future work will focus on new approaches to extend the lifetime of both nodes and connections by combining the sleeping mode with new power-aware mechanisms.

\section{References}

1. Internet Engineering Task Force, "Manet working group charter", http://www.ietf.org/html.charters/manet-charter.html.

2. C.-K. Toh "Maximum Battery Life Routing to Support Ubiquitous Mobile Computing in Wireless Ad Hoc Networks", in IEEE Communication Magazine, June 2001

3. E.Royer and C.-K. Toh, "A Review of Current Routing Protocols for Ad Hoc Mobile Wireless Networks", IEEE Personal Communications Magazine, Vol. 6, No. 2, April 1999.

4. C-K. Toh, "Associativity Based Routing For Ad Hoc Mobile Networks", Wireless Personal Comm. Journal, Special Issue on Mobile Networking and Computing Systems, Vol. 4, No. 2, Mar. 1997.

5. Z.J. Haas, "A New Routing Protocol for the Reconfigurable Wireless Networks", Proceedings of the ICUPC'97, San Diego, CA, Oct. 1997.

6. V.D. Park, M.S. Corson, "A Highly Adaptive Distributed Routing Algorithm for Mobile Wireless Networks", IEEE INFOCOM'97, Kobe, Japan, 1997.

7. C.E. Perkins, E.M. Royer, "Ad-hoc On-Demand Distance Vector Routing", Proceedings of the 2nd IEEE Workshop on Mobile Computing Systems and Applications, Feb. 1999.

8. D. Dube, C.D. Rais, K-Y. Wang, K. Tripathi, "Signal Stability-Based Adaptive Routing (SSA) for Ad hoc Mobile Networks", IEEE Personal Comm., pp. 36-45, Feb.,1997.

9. David B. Johnson, David A.Maltz, Yih-Chun Hu, and Jorjeta G. Jetcheva, "The Dynamic Source Routing Protocol for Mobile Ad Hoc Networks DSR)", Internet Draft, February 2002.

10. P.Jacquet, P.Muhlethaler, T.Clausen, A.Laouiti, A.Qayyum, L.Viennot, "Optimized Link State Routing Protocol for Ad Hoc Networks"

11. K. Fall and K. Varadhan, (Eds.) "ns Notes and Documents", The VINT Project. UC Berkeley, LBL, USC/ISI, and Xerox PARC, February 25, 2000. Available from http://www.isi.edu/ salehi/ns_doc/.

12. Laura Feeney and M. Nilsson, "Investigating the Energy Consumption of a Wireless Network Interface in an Ad Hoc Networking Environment," IEEE INFOCOM, 2001. 